\title{
A Performance Analysis on Swarm Drone Loco Positioning System for Time Difference of Arrival Protocol
}

\author{
Timothy Scott Chu, Alvin Chua, Edwin Sybingco, Ma. Antonette Roque
}

\begin{abstract}
There is an increasing application potential in swarm technology, accuracy in localization becomes a critical factor in the system in executing the desired task. As there are various localization techniques, this paper focuses on analyzing the performance of a particular radio localization technique called the Loco Positioning System operating on the Time Difference of Arrival protocol subjected to various setup configurations. The research starts with the design of various setup configurations are based on two independent parameters which are, number of anchors, and distance between anchors as they prominently affect the accuracy of the system. Position estimates are obtained by manually moving the Crazyflie equipped with a Tag within a grid system and the values are reflected through the PC client. The position estimates are then compared to the true values to obtain a relative error which is used to define the performance of the system. Data showed that operating on 4 anchors offers relatively low error and increasing the number of anchors to 8 significantly improves the accuracy of the system. Additionally, increasing the distance between anchors from $0.6 \mathrm{~m}$ to $2 \mathrm{~m}$ also improves the accuracy in the system. The obtained data offers a clear relationship between accuracy and the mentioned parameters, and a good combination of both parameters also affects the accuracy of the system. This paper offers both recommended applications and data which will aid users of the loco positioning system in determining the optimal setup based on their set of constraints.
\end{abstract}

Keywords : Loco Positioning System, Radio Localization, Swarm Drone, Time Difference of Arrival

\section{INTRODUCTION}

As UAV technology experiences rapid innovative developments, the application field is also widening up. Some of these applications may involve terrain scanning, package delivery, swarming, entertainment purposes, etc. [1]. Focusing on particularly on UAV swarming offers several possible studies as many considerations, whether internal or

Revised Manuscript Received on December 30, 2019.

* Correspondence Author

CHU, Timothy Scott*, Department of Mechanical Engineering, De La Salle University, 2401 Taft Ave, Malate, Manila, 1004 Philippines, E-mail: timothy_scott_chu@dlsu.edu.ph

CHUA, Alvin, Department of Mechanical Engineering, De La Salle University, 2401 Taft Ave, Malate, Manila, 1004 Philippines, E-mail: alvin.chua@dlsu.edu.ph

SYBINGCO, Edwin, Department of Electronics and Communications Engineering De La Salle University, 2401 Taft Ave, Manila, 1004 Philippines, E-mail: edwin.sybingco@dlsu.edu.ph

ROQUE, Maria Antonette, Department of Electronics and Communications Engineering De La Salle University, 2401 Taft Ave, Manila, 1004 Philippines, E-mail antonette.roque@dlsu.edu.ph

(C) The Authors. Published by Blue Eyes Intelligence Engineering and Sciences Publication (BEIESP). This is an open access article under the CC BY-NC-ND license (http://creativecommons.org/licenses/by-nc-nd/4.0/) external, play a role in the success of a swarm system. Swarming is the utilization of more than one aerial drone that may be programmed to operate simultaneously and autonomously or be remotely operated by a controller. This system is particularly popular with users as it resolves some of the present limitations for single drone operation, one of which is the operation period [2]. One important factor for swarm application is the capability of drones to communicate with each other and autonomously adapt to situations as the system executes a certain set of tasks [3]. Another important factor in swarming is localizing the position for each drone. The extraction of position data, in either XYZ coordinates or geographic coordinates, from an object of interest by using external sensors or components is called localization. External components such as satellites, vision cameras, radio signals, etc. are the current developments that are utilized for the detection of aerial drones and return a point, represented in coordinate systems to show the drone's position from a given reference. The Global Positioning System (GPS) returns values in longitudes, latitudes, and elevation parameters; however, this system is limited to mainly outdoor applications. Inaccuracies in readings or estimates occur in the presence of GPS denied environments or indoor applications. Indoor localization techniques were developed as one solution to the mentioned limitation of GPS; and, one of these localization techniques is executed by utilizing several vision cameras [4] strategically positioned to capture the drone at different angles during operation. This system can accurately estimate the position of each drone, which can be further used for various applications. J. Priess et al conducted and documented a study in [5], utilized multiple Crazyflies and used these drone units in swarm applications. A total of 49 units were used and termed the entire system as the Crazyswarm. The researchers focused on vision localization by utilizing motion capture technology, specifically the Vicon Tracker. Reflective markers should be placed on the drone unit, a required method for the Vicon Tracker, and the camera captures the movements of the drone to obtain the raw position data. Raw data coupled with the Iterative Closest Point (ICP) algorithm, allows the system to record the movement for each marker of the drone and offers a frame per frame comparison. This leads to a comparison of each successive image for the whole duration of the experiment, obtaining a position estimate data. Additionally, researchers applied a program wherein each drone is capable of detecting its proximity with other drone units and autonomously make adjustments to avoid collisions. 
Radio localization is another indoor localization technique that utilizes radio signals. The process involves 2 main components, namely the anchor and the tag. Anchors are external components, which number to at least 3 to 4 units, and are positioned strategically to create a controlled flight space. The tag serves as the object of interest and is mounted actively communicates with the tag through data packets transmitted through radio signals; consequently, determining the estimated position of the drone. Literature resources on radio localization for aerial drone applications are currently still few since this localization technique is a newly developed application.

The used of Ultra-Wideband radios are particularly popular. This prompted researchers from the University of California, Berkley to study localization effectivity utilizing ultra-wideband (UWB) radios and propose a way to improve it. The proponents focused on developing a generic extended Kalman filter (EKF) which aimed to collate data from the on-board IMU and UWB radios and integrate both sets of data to offer a more accurate estimation. The experiment process involved conducting test flights such as hovering and executing a circular trajectory of a single drone and obtaining position estimates as the drone executes the mentioned flight tests. The research used a motion capture system as the base data for comparison with the estimated data obtained through 5 UWB beacons. After the experimentation, it was observed that the position tracking error was relatively large [6].

For localization applications, accuracy is a very important variable, thus various studies were conducted to develop efficient techniques which aim to increase the accuracy in a system. Researchers from De La Salle University-Manila developed a modified Sliding Mode Control (SMC) method which was implemented in quadrotors. The method is non-linear and it translates a system's dynamics to a designated set of trajectories; and, this method was validated through comparing the data between the developed method and the PID controller, which were generated from experiments. It was observed that the SMC method was able to generate more accurate readings for roll, pitch, and yaw compared to the designated PID controller [7].

The same proponents from the University of California offered a different approach to radio localization and developed an EKF estimator that can accompany a mobile anchor into the system. This was primarily achieved through minimizing the determinant of the covariance matrix. The test environment and experimentation are similar to the setup used in [6]; however, the researchers added a dynamic anchor into the setup. After the experiment, trajectory tracking showed an improvement of $14 \%$ in the obtained results [8].

The Crazyflie can accompany additional components which are mounted on the drone, one of these expandable systems is the Loco Positioning System. The mentioned system operates on the concept of radio localization and particularly uses ultra-wideband (UWB) radios. The system possesses two protocols which are the Two-Way Ranging protocol and the Time Difference of Arrival, both will be further explained in the succeeding section. Current resources on the performance of the Loco Positioning System do not offer much data on the accuracy of various anchor configuration setup regarding the number of anchors used on to the drone. The anchors are used as reference points as it

and the distances in between anchors.

The paper presents a performance analysis of loco positioning systems using the Time Difference of Arrival protocol as it is more suitable for swarm applications. In addition to this, the methodology presents the various setup configurations utilized in the experimentation of the system to obtain necessary data for analysis. The data are obtained to extract the percentage of error derived from each setup which is represented in figures and percentages. After this, a performance analysis is done based on the obtained data which presents values that will aid in determining the ideal setup based on the accuracy and the given constraints on the number of anchors, and the space between anchors in the setup. Recommended applications are also offered in the paper for each configuration which will greatly also aid users of the mentioned protocol of the Loco Positioning System. Users may use this paper as a reference in determining the optimal setup with considerations on the desired margin of error, size of available flight space, and the number of anchors to be used.

\section{LOCO POSITIONING SYSTEM (LPS)}

The Loco Positioning System is a tool that operates on the concept of radio localization using 2 main components, namely the anchor and tag. Multiple anchors or the Loco Positioning Anchors are separate components used to set the boundary or flight environment, while the tag or Loco Positioning Deck is mounted on the Crazyflie to serve as the object of interest. The estimated position of the Crazyflie, with a tag, is obtained through radio communication between anchor and tag. A complete system is usually comprised of at least 3 or 4 anchors positioned at a specified location and distance from each other. Fig. 1 shows the general diagram of the LPS setup. The algorithm of the system is integrated with data fusion filter algorithms such as the Kalman filter, to collate relevant data and provide an accurate estimate localization of the object of interest. Anchor and decks are equipped with UWB radios which are based on the Decawave DWM 1000 module. Two protocols such as the Two-Way Ranging (TWR) and the Time Difference of Arrival (TDoA) are available in the LPS [9]. However, this research covers only experimentations done in the Time Difference of Arrival protocol.

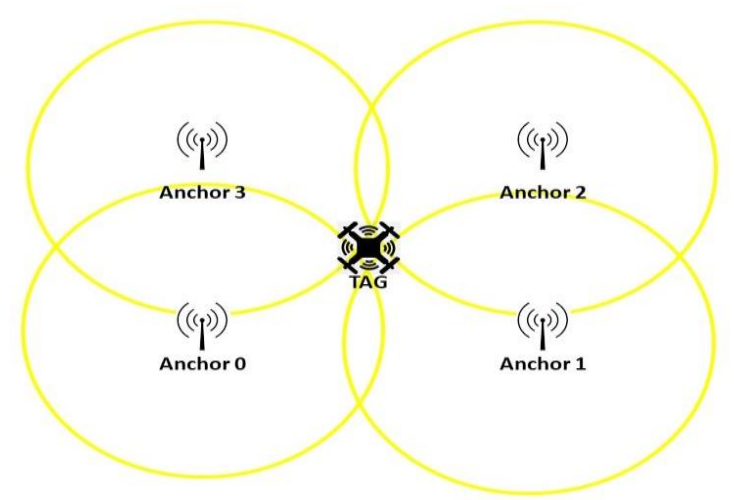

Fig. 1. Loco Positioning System

Published By:

Blue Eyes Intelligence Engineering 


\section{A. Two-Way Ranging (TWR) Protocol}

The TWR protocol is one of the ranging algorithms present in the Loco Positioning System. The algorithm utilizes a tag and an anchor which are in constant communication with each other through a process of transmitting, receiving, and replying data packets in a particular order. The TWR protocol initiates when the tag transmits a data packet to the anchor, and a reply from anchor to tag follows, establishing 1 exchange. Another exchange is executed to diminish the clock drift error and to create reliable feedback, thus the total process requires 2 exchanges.

\section{B. Time Difference of Arrival (TDoA) Protocol}

The TDoA protocol has 2 modes but with a similar function. It utilizes 2 components namely the anchor, serving as the reference point, and the tag which is mounted on the Crazyflie. In this protocol, the anchors are continuously sending data packets while the tag is actively receiving these packets and the distance is measured by computing the time difference of arrival of the data packets. In this protocol, since the tags are tasked to receive data packets only, it offers less computational load as compared to the Two-Way Ranging (TWR) protocol making it the better protocol for swarming. Fig. 2 shows the general process diagram of the TDoA protocol. Generally, the TDoA can be calculated with the difference between $\Delta \mathrm{Tx}$ and $\Delta \mathrm{Rx}$, and the data is then received on the last data packet (E3) from the anchor. $\Delta \mathrm{Rx}$ can be calculated when the data packets from anchors 0 and 1 are obtained, specifically at exchanges 2 and 3 (E2 and E3). Meanwhile, $\Delta \mathrm{Tx}$ is computed on anchor 1 , provided that the time it takes to send a message from anchor 0 to anchor 1 is known. The required value can be obtained since both anchors are communicating with each other through the TWR protocol. Corrective factors are also applied to minimize clock drift errors. In Fig. 3, the consecutive packet data from anchor 1, E1 and E3, are used to calculate a corrective factor (C.F.) by using (1) while the general equation to obtain the TDoA showin in (2) [10].

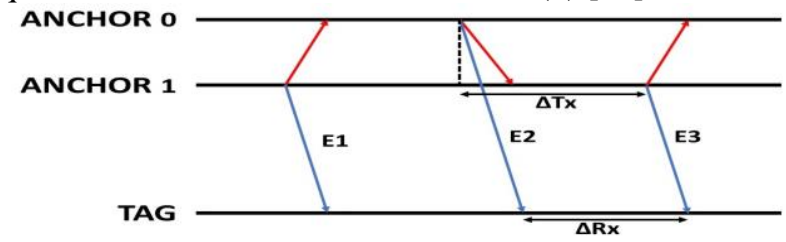

Fig. 2. General TDoA Protocol Operation

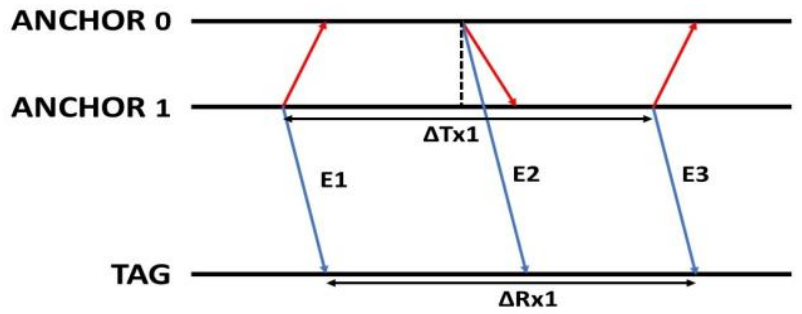

Fig. 3. TDoA Corrective Factor Computation

$$
\begin{gathered}
\text { C.F. }=(\triangle R x 1) /(\triangle T x 1) \\
T D o A=\Delta R x-(C . F . \times \triangle T x)
\end{gathered}
$$

The Time Difference of Arrival Protocol 2 (TDoA 2) is a subsegment of the TDoA protocol and is more restrictive than the TWR protocol in terms of the flight environment. This protocol works best when the tag is always within the set boundary. This offers the assumption that this protocol works best when utilizing 8 anchors position on the corners of the flight environment; however, since the protocol is time-slotted, the number of utilized anchors is limited to 8 . Furthermore, the accuracy of this protocol is close to the TWR protocol [12].

Time Difference of Arrival Protocol 3 (TDoA 3) on the other hand may possess various similarities with the TDoA 2, it is capable of supporting multiple tags and anchors. This protocol randomizes the time it transmits data unlike TDoA 2 wherein it follows a specific schedule, thus allowing the accompaniment of more than 8 anchors into the system. Additionally, the system is capable of manage loss or addition of anchors dynamically, allowing the system to still function. More anchors would allow users to set a larger flight environment or even through barriers which limit the line of sight between anchors. However, the accuracy of the data obtained in this protocol may vary a little compared to the TDoA 2 protocol [11].

\section{Time of Flight (ToF)}

One of the key concepts utilized for the TDoA protocol is the Time of Flight or TOF. The process begins by transmitting a data packet from ANCHOR to TAG and a reply is sent after a specified time interval. With this information, a total roundtrip time is obtained which can be multiplied to the speed of light, $C$. The product of the roundtrip time and the speed of light is the round-trip distance traveled which is halved to obtain the distance between the tag and the anchor. The equation presented in (3) shows the numerical relationship for the computation of the distance between tag and anchor [12].

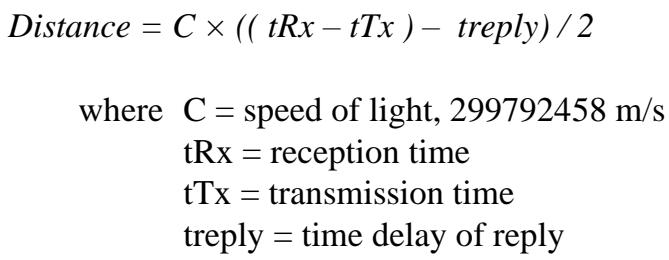

\section{MATERIALS}

The aerial drone which will be utilized in this study is the Crazyflie as shown in Fig. 4, which is developed by Bitcraze. It is a robust drone that is equipped with the bare minimum for its operation to lessen costs. The drone is 4 inches by 7 inches and weighs about 27 grams, and is designed with growing developments in mind. Additionally, due to the drone's size and weight, it poses a small momentum force which would be relatively harmless if the unit crashes to a living being. Expansion systems, which are mountable to the unit, are also developed to allow more applications for the Crazyflie drone. The most important factor is that the Crazyflie is an open-source drone that allows the researchers to program the drone to execute a specified task [13]. Also, the components of the Loco Positioning System, such as the Loco Positioning Node, and Loco Positioning Deck, as shown in Fig.

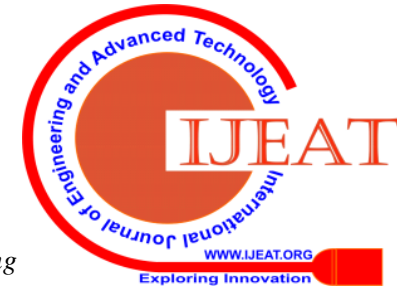


5 and Fig. 6 respectively, will be utilized in this research. The number of nodes, serving as the anchors, used will vary for each configuration.

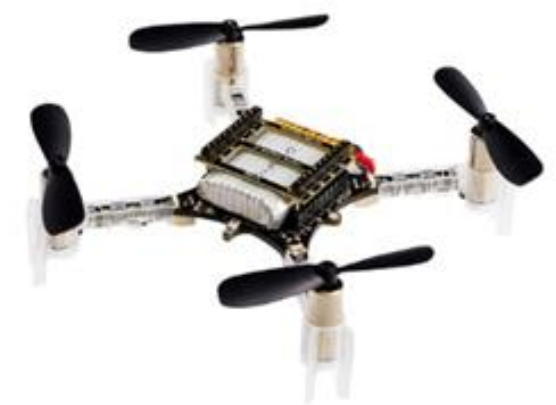

Fig. 4 Crazyflie 2.1, Retrieved from [13]

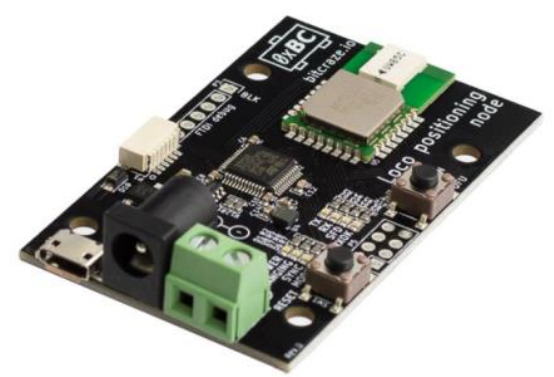

Fig. 5. Loco Positioning Node, Retrieved from [14]

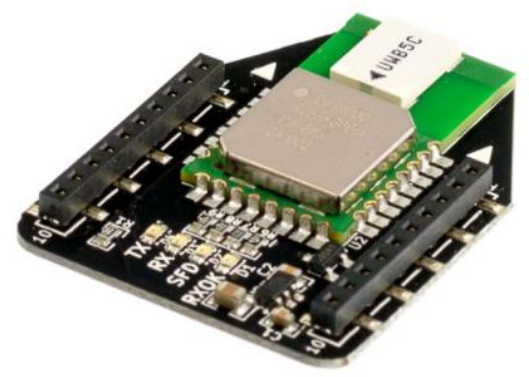

Fig. 6. Loco Positioning Deck, Retrieved from [15]

\section{METHODOLOGY}

Fig. 7 shows the experimental process utilized in this study. It can be defined into 3 steps, the Design or Definition of Different Cases, Setup of the Cases, and Position Experiment for the specified cases. Further explanation of each process is explained in the latter sections.

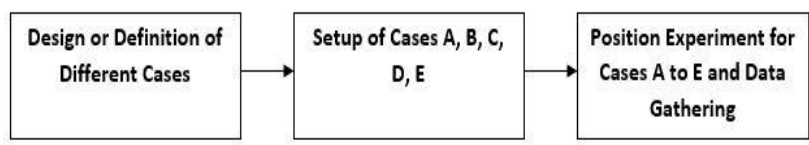

Fig. 7. Methodology Flowchart

\section{A. Design/Define Different Cases}

Experiments are conducted to various configurations, serving as case setups, to observe and analyze the behavior and the accuracy of the TDoA protocol in the Loco Positioning System. The configurations are set up based on two main categories, namely, the quantity of the utilized anchors, and the space set in-between anchors with 8 anchors.
1) Case Setup Based on Number of Anchors

This category is set to determine the optimal number of anchors required to get the desired accuracy, and the latter configuration is set to determine the optimal distance between anchors, also based on a set allowable margin of error. Each category offers 3 different setups for comparison. The setup is set to have a grid system, generally with $0.3 \mathrm{~m}$ increments unless specified, serves as the true data and the reference points to where the Crazyflie, equipped with the TAG, is placed. Estimated data reading is obtained from the Crazyflie PC Client, which is the official software for the Crazyflie drone. The PC client possesses an LPS functionality tab which can return estimates on $\mathrm{X}, \mathrm{Y}$, and $\mathrm{Z}$ coordinates on each position the aerial drone is set. The experiment is done in a 2D space; however, similar behavior should be observed as well when applying in a 3D setup.

For the category of case setup based on number of anchors, 3 configurations based on the number of anchors, are presented. Data such as the average relative errors will be obtained from these configurations to determine the performance for each case.

Case A utilizes 8 anchors, as recommended by the developers, with a distance of 1 meter along the $\mathrm{x}$-axis and 0.6 meters along the $y$-axis which forms a rectangular space. Fig. 8 offers a visualization of this configuration.

\section{ANCHOR PLACEMENT} (METERS)

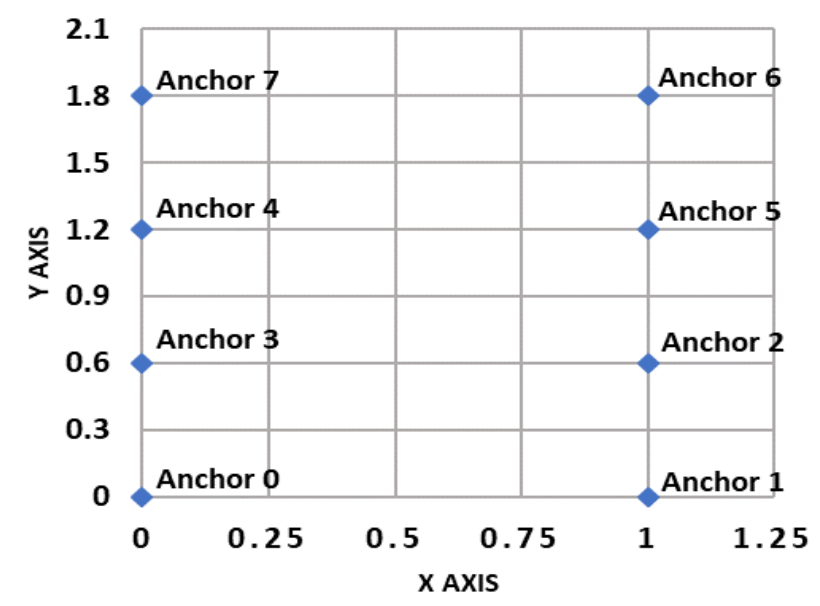

Fig. 8. Case A - 8 Anchors

Fig. 9 shows the configuration of case B, which utilizes 6 anchors. The specification on the distances is the same with case $\mathrm{A}$. This case aims to validate the operation of the TDoA protocol with 6 anchors, which is the minimum required. 
ANCHOR PLACEMENT (METERS)

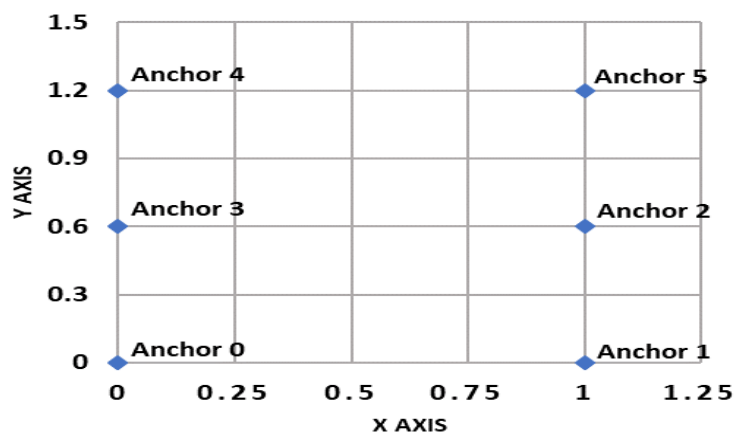

Fig. 9. Case B - 6 Anchors

Finally, for this category, case C utilizing 4 anchors to form a square space as visualized in Fig. 10 is tested to observe the accuracy of the data when the anchors are less than the required.

\section{ANCHOR PLACEMENT (METERS)}

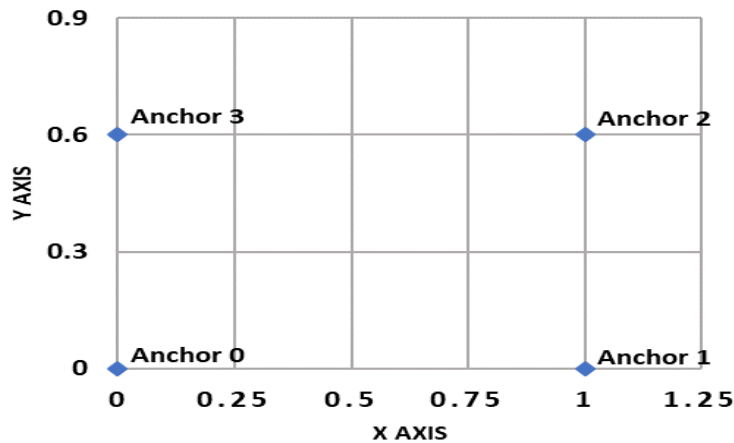

Fig. 10. Case C - 4 Anchors

2) Case Setup Based on Distance Between Anchors

Three more cases are presented under this category, utilizing 8 anchors, this portion of the study aims to observe the behavior and the accuracy of the data through the different setup on the distance. This portion will use the same parameter of average relative errors as the criteria in determining the performance of each configuration.

Case D, as shown in Fig. 11 is configured to set the anchors 2 meters from each other along the $\mathrm{x}$-axis, and 1 meter along the $y$-axis, with increments of $0.6 \mathrm{~m}$ for the specified points. Ideally, a bigger space would offer better accuracy; hence, this case aims to validate the mentioned claim.

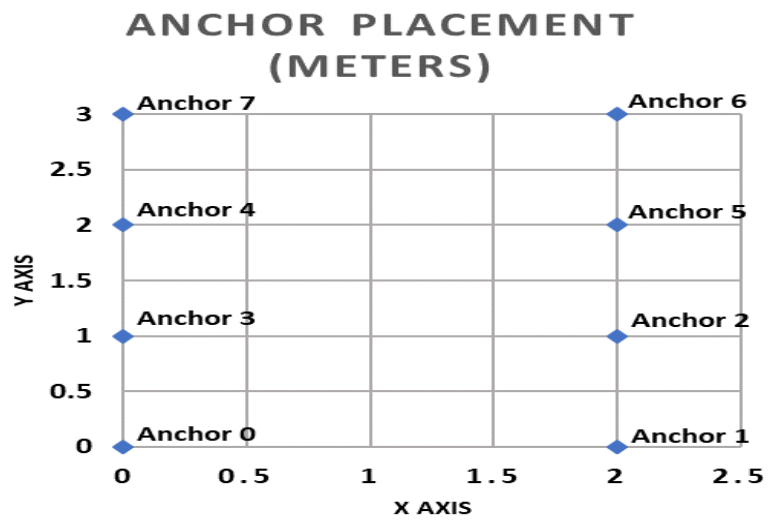

Fig. 11. Case D - 8 Anchors (2m Apart)
Case A will also be utilized in this category as it also meets the criteria specified in this set.

Fig. 12 is the setup used to test the functionality of the TDoA protocol under space constraints. The setup is set to have a $0.6 \mathrm{~m}$ distance between anchors both in the $\mathrm{x}$ and $\mathrm{y}$ axes and increments of the specified points are set to be $0.15 \mathrm{~m}$.

ANCHOR PLACEMENT (METERS)

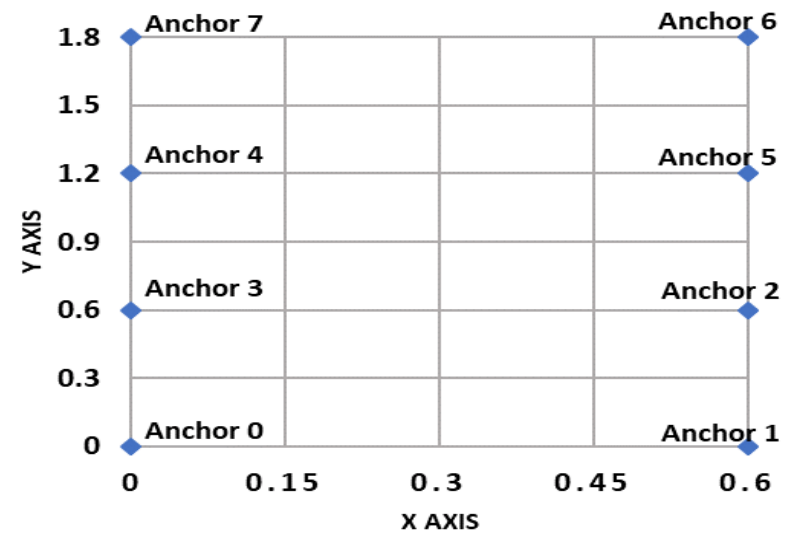

Fig. 12. Case E - 8 Anchors (0.6m Apart)

\section{B. Setup for Each Case}

Anchors are labeled from 0 to 7 and are utilized respectively as per the requirements for each case. Coordinates in meters will be written and recorded to the anchors through the PC client. Anchor 0 will be the reference point, with coordinates of $0,0,0$. The anchors are then set into the specified configuration of the case and are powered up by power banks. To ensure that the active protocol of the anchor is TDoA, an LED indicator, particularly the power LED should be lit only. The Crazyflie then is switched on by connecting the power cables; additional configuration, the Crazyflie should face the positive $\mathrm{X}$-axis and should be inside the boundary set by the anchors for the calibration of its onboard inertial measurement unit (IMU). To verify the operation of the system, the PC client should reflect the anchors and the tag and trace the tag's movement in the graphs in the client.

\section{Position Experiment for Each Case}

The experimentation primarily involves manually moving and positioning the aerial drone along with the points inside the set grid for each case. After this, the $\mathrm{x}$ and $\mathrm{y}$ estimates are recorded, and the experiment is conducted again for around 3 to 5 times. An average data is then obtained which will be compared to the true values set by the grid. Fig. 13, Position Experiment Setup, is a visual representation of the experiment process. The data gathered will then be analyzed and evaluated; and, it should show data that are close to the true values of the setup. Additionally, experimentation will be conducted for both TDoA 2 and TDoA 3 protocols. 


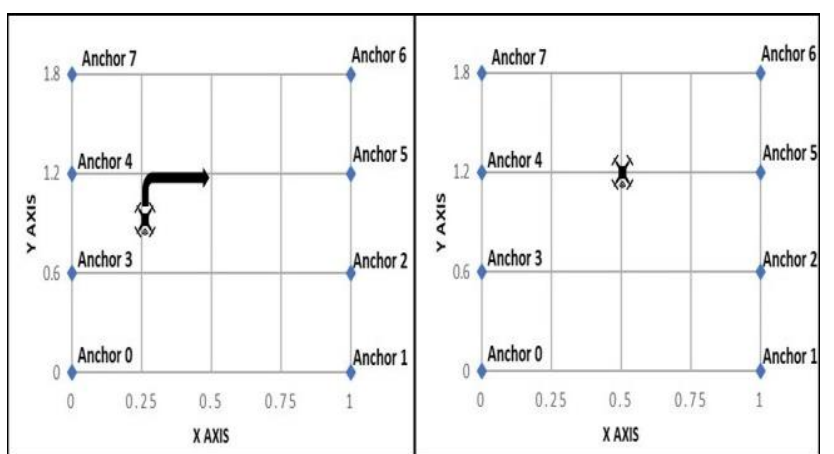

Fig. 13. Position Experiment

\section{RESULTS AND DISCUSSIONS}

Results for each case is presented with a figure showing shapes with varying colors in a $2 \mathrm{D}$ graph. Anchor location is denoted with a yellow triangle, while the specified position and the estimated positions are represented with blue squares and green diamonds respectively. Furthermore, data labels such as set 1 , set 2 , set 3 , set 4 , and set 5 are also available and positioned beside the symbol which denotes estimated position. This allows a clearer representation of where the point should be located in comparison to the blue squares (specified coordinates); consequently, this makes data comparison easier. Set 1 refers to the column of blue squares (specified coordinates) nearest to the reference point, eg. Case A Set 1 refers to the set of points on $\mathrm{X}=0.0 \mathrm{~m}$. Succeeding sets correspond to the succeeding columns on the right after the first set.

\section{A. Case Setup Based on Number of Anchors}

Because This portion shows the results obtained for configurations based on the number of anchors.

1) Case $A$

On the TDoA 2 protocol, represented by Fig. 14, most of the estimated points are close to their respective true values, forming a linear pattern. Set 2, 2 points have variances of $0.19 \mathrm{~m}$ and $0.12 \mathrm{~m}$, while all the other points have variances that are less than or equal to $0.05 \mathrm{~m}$. The average relative error obtained in this setup is $6.58 \%$.

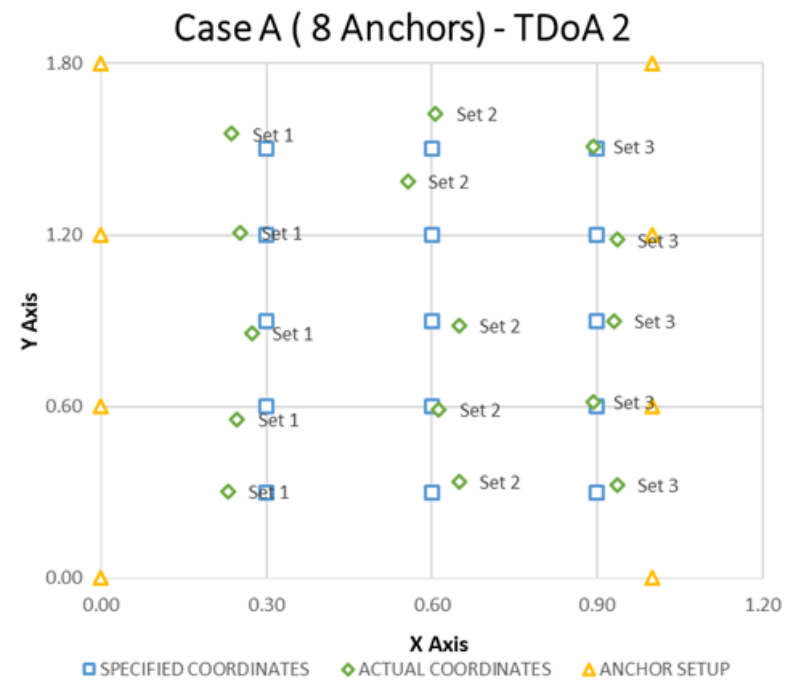

Fig. 14. Case A Results (TDoA 2)

On TDoA 3 protocol it possessed similar behavior to the results of the TDoA 2 Case A. It possesses a maximum variance of $0.15 \mathrm{~m}$, followed by $0.12 \mathrm{~m}$ in set 3 and set 1 respectively; meanwhile, most of the other points have variances less than $0.06 \mathrm{~m}$. The error on this protocol with the same setup is $6.83 \%$, slightly more than its TDoA 2 counterpart. Fig. 15 shows the data set of this case.

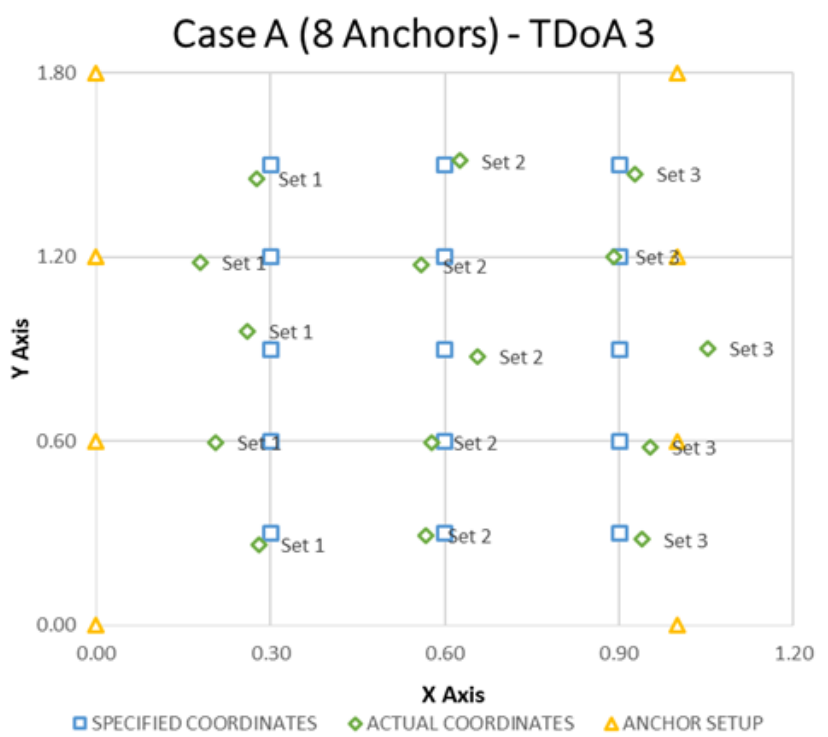

Fig. 15. Case A Results (TDoA 3)

\section{2) Case $B$}

Results for TDoA 2 protocol as seen in Fig. 16, shows that the estimated data, particularly the second point in set 3 , are far from the true value. The mentioned point had a $0.2 \mathrm{~m}$ variance, while half of the remaining points have variances from $0.7 \mathrm{~m}$ to $0.1 \mathrm{~m}$. The setup gave an average relative error of $10.98 \%$.

Meanwhile, Fig. 17 shows the results for the TDoA 3 protocol showed the same behavior when the first point in set 3 possessed also a variance of $0.2 \mathrm{~m}$. However, the setup is slightly more accurate than the TDoA 2 counterpart, having an average relative error of $10.38 \%$.

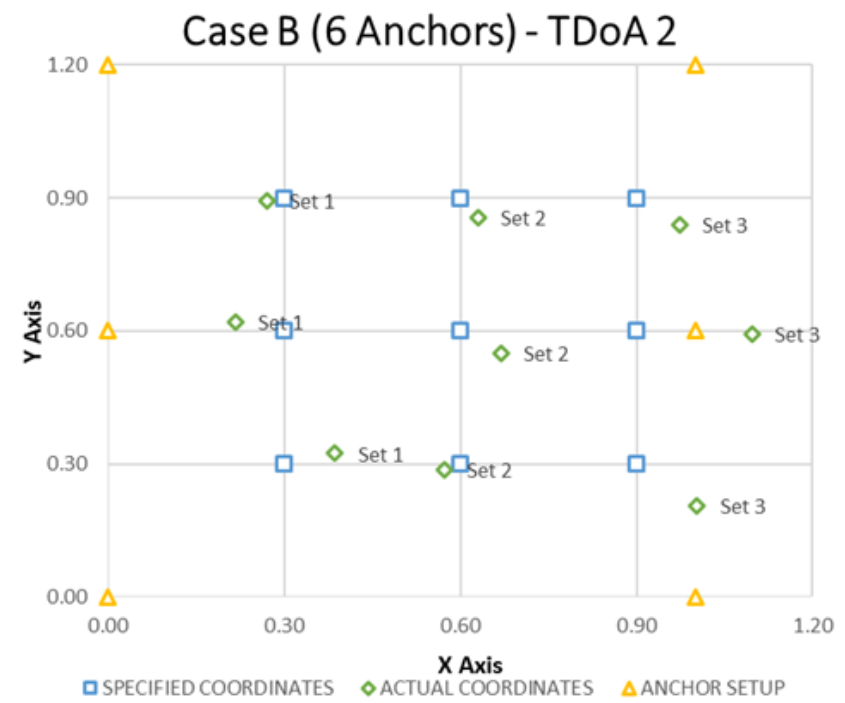

Fig. 16. Case B Results (TDoA 2)

3) Case $C$ :

Published By:

Blue Eyes Intelligence Engineering 
Utilizing 4 anchors and 3 specified points, the TDoA 2 protocol gave an error of $12.38 \%$ with a maximum variance of $0.18 \mathrm{~m}$.

TDoA 3 protocol, given the same circumstances, gave better results with an average relative error of $10.37 \%$. The maximum variance this protocol experienced with the setup is $0.15 \mathrm{~m}$. Fig. 18 and 19 show the results from TDoA 2 and TDoA 3 respectively.

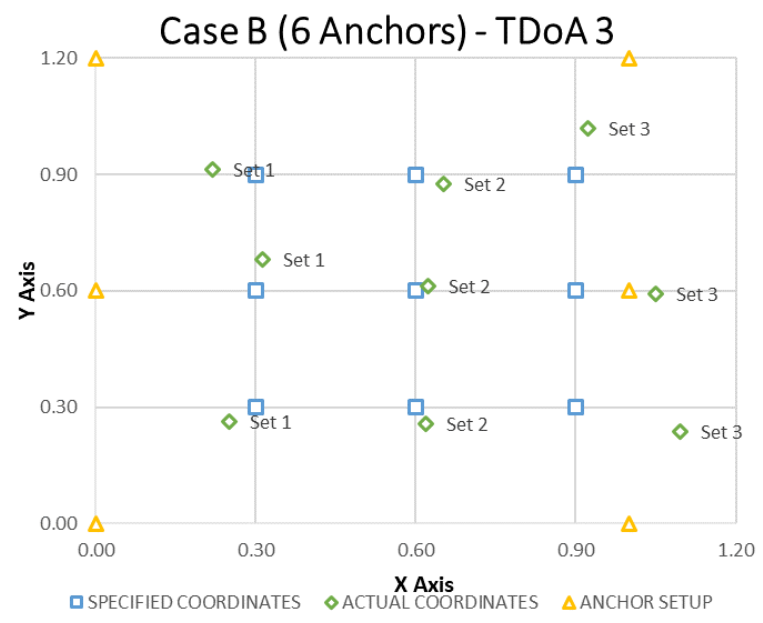

Fig. 17. Case B Results (TDoA 3)

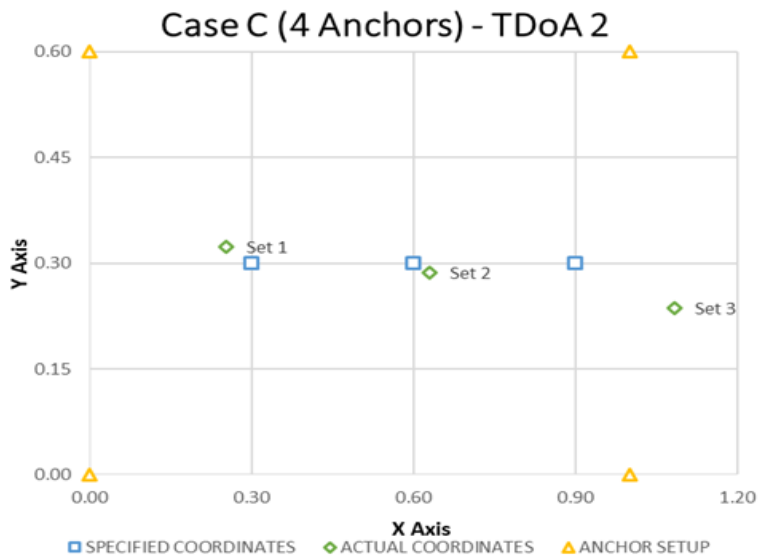

Fig. 18. Case C Results (TDoA 2)

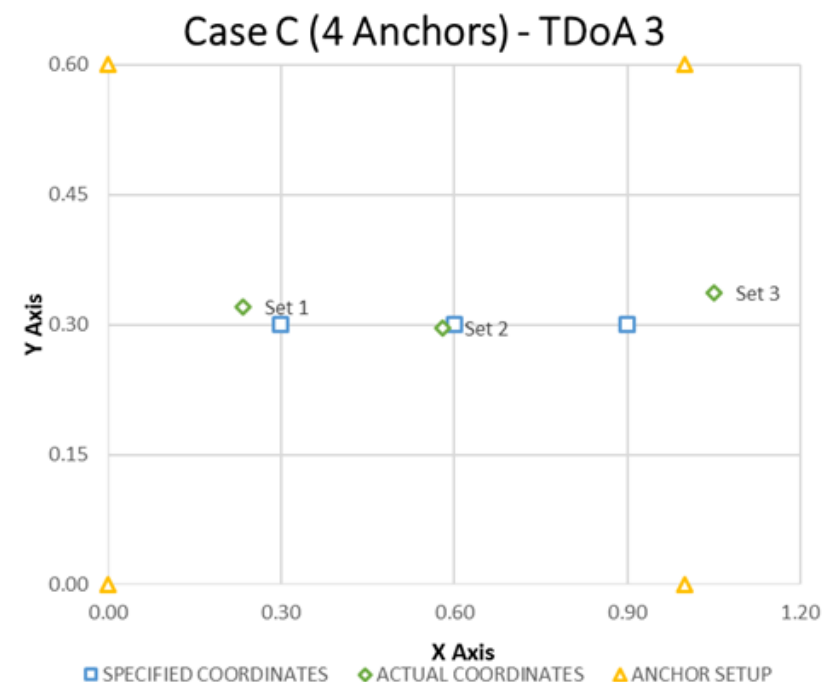

Fig. 19. Case C Results (TDoA 3)

\section{B. Case Setup Based on Distance Between Anchors}

This portion shows the results obtained for configurations based on the number of anchors

1) Case $D$

This configuration utilized 8 anchors with a large operational space and the specified positions which are set to have increments of $0.6 \mathrm{~m}$ from each other as shown in Fig. 20 . The findings for the setup show that an ideal setup would have similar configurations. Average relative errors of $2.89 \%$ for TDoA 2 and $2.63 \%$ for TDoA 3 were obtained in the experiment. For the TDoA 2 protocol, the observed variance peaks at $0.06 \mathrm{~m}$ on 3 points while most points showed a variance of $0.03 \mathrm{~m}$.

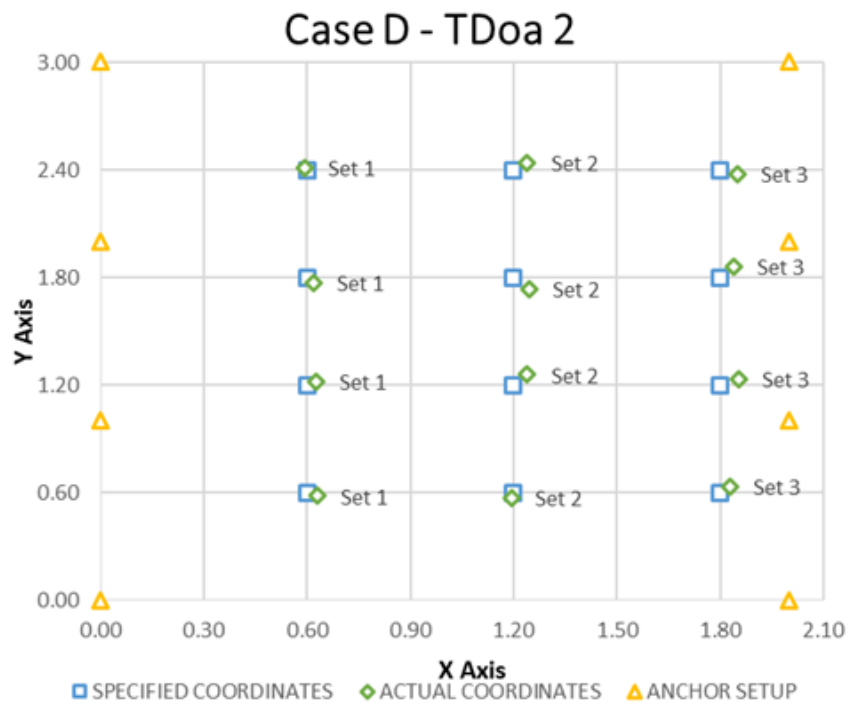

Fig. 20. Case C Results (TDoA 3)

On TDoA 3 protocol showed the same behavior as of the TDoA 2 protocol wherein the maximum variance is $0.6 \mathrm{~m}$ on one point while most points experience a variance of $0.3 \mathrm{~m}$. The results are represented in Fig. 21.

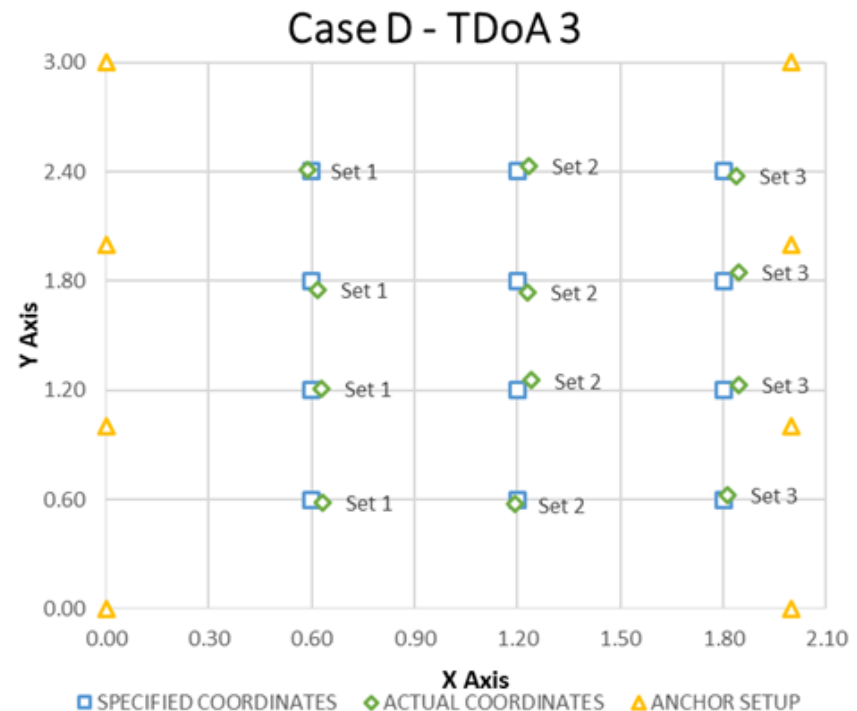

Fig. 21. Case D Results (TDoA 3) 


\section{2) Case $E$}

Case $\mathrm{E}$ is set with 8 anchors placed $0.6 \mathrm{~m}$ apart from each other and the increments of the specified positions are $0.15 \mathrm{~m}$ from each other. The TDoA 2 protocol was not able to operate properly on this setup as it gave random readings for the estimated position of the tag. Thus, the data of this protocol in this configuration is unobservable in this experiment, resulting in the absence of a graph.

TDoA 3 protocol, however, was able to function in this configuration but gave off inaccurate estimates. Fig. 22, shows that there were no clear patterns formed with the estimated data. The error obtained in this setup is $15.39 \%$. The setup possessed points with large variances such as 0.26 $\mathrm{m}, 0.22 \mathrm{~m}$, and $0.19 \mathrm{~m}$.

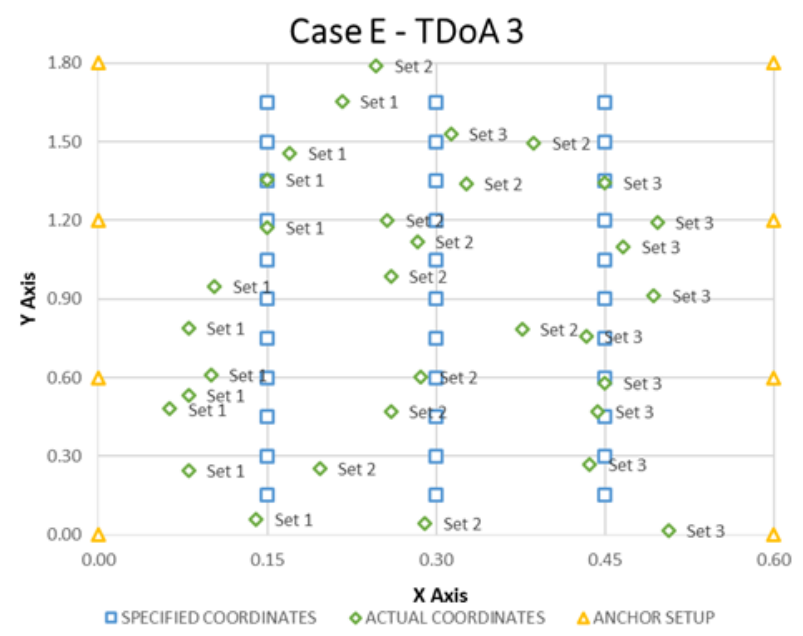

Fig. 22. Case E Results (TDoA 3)

\section{Discussion}

Case A validated that the two protocols will give similar accuracy. TDoA 2 gave an average relative error of $6.58 \%$ while TDoA 3 gave $6.83 \%$. This validated the claim made in the framework that the data in TDoA 3 may vary a little with TDoA 2, however, it is still relatively accurate.

Case $B$ sets the 2 protocols to operate with the minimum number of anchors recommended for this setup; as expected, TDoA 2 and TDoA 3 are capable of functioning in this setup with average relative errors of $10.98 \%$ and $10.38 \%$ respectively. Though it can be observed in this setup that removing 2 anchors from the previous setup increases the errors by around $4 \%$.

The configuration in case $\mathrm{C}$ has 2 fewer anchors from the developer's recommendation. The objective of this case is to determine the functionality of the protocols in this setup. Both protocols were able to function well, however, TDoA 2 retuned an average relative error of $12.38 \%$ while TDoA 3 returned an average relative error of $10.37 \%$. Since the TDoA 3 protocol is capable of adapting to the number of anchors, it is able to return data with similar average relative errors. The TDoA 2 protocol, on the other hand, gave around 2\% more error compared to case B.

Further experimentation was done on case $\mathrm{C}$, increasing the $\mathrm{y}$-axis distance from $0.6 \mathrm{~m}$ to $1 \mathrm{~m}$, to accommodate more specified points for comparison, having 9 points instead of 3 . The setup and results for TDoA 2 and TDoA 3 are shown in Fig. 23 and 24 respectively. The experiment validated the results of the original setup when it returned an average relative error of $12.91 \%$ for TDoA 2 and $10.45 \%$ for TDoA 3 .

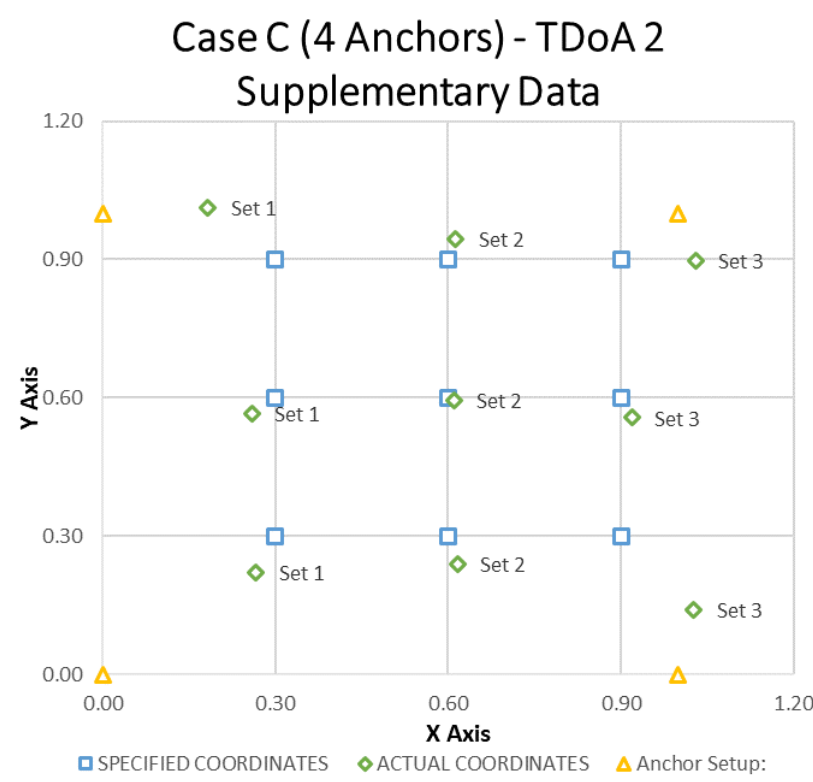

Fig. 23. Case C Supplementary Results (TDoA 2)

Cases $\mathrm{A}$ to $\mathrm{C}$ presented the behavior or the accuracy concerning the number of anchors present in the system. Generally, the accuracy of the system is directly proportional to the number of anchors in the system. This suggests that utilizing more anchors increases the accuracy of the system as it decreases the error obtained. Table 1 shows a summary of the data obtained from setups based on the number of anchors. This will aid users in determining how many anchors will be utilized for their system to be optimal given their constraints and their desired allowable error.

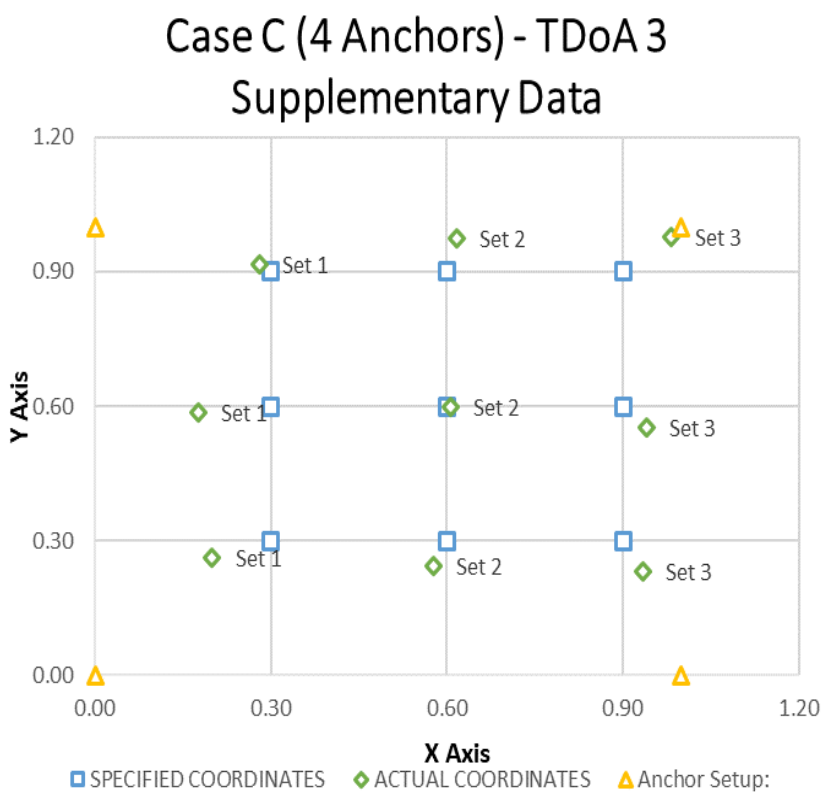

Fig. 24. Case C Supplementary Results (TDoA 3) 
TABLE- I: Summary Of Results For Cases Based On Number Of Anchors

\begin{tabular}{|l|c|c|c|}
\hline \multirow{2}{*}{ Case } & Qty of Anchors & \multicolumn{2}{|c|}{ Average Relative Error } \\
\cline { 3 - 4 } & & TDoA 2 & TDoA 3 \\
\hline Case A & 8 & $6.58 \%$ & $6.83 \%$ \\
\hline Case B & 6 & $10.98 \%$ & $10.38 \%$ \\
\hline Case C & 4 & $12.38 \%$ & $10.37 \%$ \\
\hline
\end{tabular}

Case $\mathrm{D}$ would be the best case to use as it offered average relative errors of $2.89 \%$ and $2.63 \%$ for TDoa 2 and TDoA 3 protocols respectively. The configuration would require a relatively large indoor space, around 3 meters by 2 meters, for operation. This indicates that the larger the working space results in a more accurate estimated data. However, the maximum space distance for LPS effectivity is not yet determined. This case also showed that a $0.6 \mathrm{~m}$ gap between anchors and specified points would return more accurate estimated data.

Case E, with a setup of $0.6 \mathrm{~m}$ distance in between anchors was found to be ineffective for the TDoA 2 protocol since data could not be obtained. The estimated data would either overshoot from the boundary or frantically moving from one point to another, causing the data to be unobservable. However, in the TDoA 3 protocol, data was obtained but possessed an average relative error of $15.39 \%$. Additionally, the estimated data points in the figure do not show a clear pattern making it not the best setup to use for swarm applications.

Cases D, A, and E showed that the accuracy of the system is also directly proportional to the distance between anchors. Table 2 shows the summary of the data obtained; as the distances between anchors increase, the accuracy also increases since error would decrease in the same phenomenon. The table offers information on the spacing users will adapt to their system to obtain a desired margin or error.

TABLE- II: Summary of Results for Cases Based on Distance Between Anchors

\begin{tabular}{|l|c|c|c|c|}
\hline \multirow{2}{*}{ Case } & \multicolumn{2}{|c|}{ Distance (m) } & \multicolumn{2}{c|}{ Average Relative Error } \\
\cline { 2 - 5 } & X Axis & Y Axis & TDoA 2 & TDoA 3 \\
\hline Case D & 2.0 & 1.0 & $2.89 \%$ & $2.63 \%$ \\
\hline Case A & 1.0 & 0.6 & $6.58 \%$ & $6.83 \%$ \\
\hline Case E & 0.6 & 0.6 & N/A & $15.39 \%$ \\
\hline
\end{tabular}

\section{CONCLUSION AND RECOMMENDATION}

The objective of the paper is to present a performance analysis of a loco positioning system that utilizes the TDoA protocol. Additionally, the mentioned protocol possesses 2 sub-protocols which are TDoA2 and TDoA3. Experimentation was done by subjecting both sub-protocols to various setup configurations with the goal of locating the manually positioned Crazyflie with tag within the grid system. The 2 main parameters which define the setup configuration are the number of anchors used, and the distance between each anchor. The output of the experimentation is the position values estimated by the experimental setup, and these values are obtained through the use of the Crazyflie PC Client. The obtained data is then compared to the baseline data which represents the true-values of the experiments to obtain the relative error. The relative error parameter was used by the researchers in order to create a performance analysis for the setup configuration.

The TDoA protocol is capable of operating with 4 anchors; though, increasing the number of anchors from 4 to 8 anchors shows that the errors for TDoA 2 protocol are nearly halved, while TDoA 3 protocol experienced a decrease in error by around $3.50 \%$. Further observations were made by comparing the behavior of both protocol in Cases B and C; it can be inferred that the TDoA 3 protocol is capable of adapting to losses in anchors since the error is maintained or shows minimal variance, while the TDoA 2 protocol is affected as the error increased by more than $1 \%$. Thus, when operating below 8 anchors, TDoA 3 is recommended since it showed more reliability in the experiments. Operation of the TDoA protocol is not recommended on a small space which results in the distance between anchors to be $0.60 \mathrm{~m}$ as it can greatly affect the accuracy of the estimated data. TDoA 2 protocol was not able to operate properly given the same conditions, and the TDoA 3 protocol an error of $15.39 \%$. Increasing the distance greatly decreases errors as shown in case $\mathrm{D}$, wherein the setup gave off errors of $2.89 \%$ and $2.63 \%$ for TDoA 2 and TDoA 3 protocol respectively.

Users may utilize the supplementary setup of Case C, 4 anchors with a 1-meter distance between anchors, in the application for single drone familiarization as it offers around $10 \%$ to $12 \%$ relative errors. Though for autonomous flight, 6 anchors will be recommended, similar to case B. This is suitable for an educational platform but should be complemented with necessary safety considerations. In applications that require high accuracy estimations such as swarming, case D setup configuration would be the ideal setup. TDoA 2 protocol would not be able to handle more than 8 anchors as specified by the developers; meanwhile, the TDoA 3 protocol can adapt accordingly and more anchors would offer redundancy and offer more accurate results. Additional studies that can be made in determining the maximum distance between anchors can be done based on accuracy to further determine additional setup configurations, however, an error of $2 \%$ to $3 \%$ is sufficient to accompany a wide variety of applications.

\section{ACKNOWLEDGMENT}

The proponents would like to extend its gratitude to the University Research Coordination Office of De La Salle University Manila, Philippines for providing support to the proponents for the conduct of the research. 


\section{REFERENCES}

1. C. Dim, F. Nabor, G. Santos, M. Schoeler, \& A. Chua, (2019, May), "Novel Experiment Design for Unmanned Aerial Vehicle Controller Performance Testing." In IOP Conference Series: Materials Science and Engineering (Vol. 533, No. 1, p. 012026), IOP Publishing.

2. Karaca, Y., Cicek, M., Tatli, O., Sahin, A., Pasli, S., Beser, M. F., \& Turedi, S. (2018). The potential use of unmanned aircraft systems (drones) in mountain search and rescue operations. The American journal of emergency medicine, 36(4), 583-588.

3. Ahmed, H.R., \& Glasgow, J.I. (2012). Swarm Intelligence : Concepts , Models and Applications Technical Report 2012-585.

4. Badshah, A., Islam, N., Shahzad, D., Jan, B., Farman, H., Khan, M., ... \& Ahmad, A. (2018). Vehicle navigation in GPS denied environment for smart cities using vision sensors. Computers, Environment and Urban Systems.

5. J. A. Preiss , W. Honig, G. S. Sukhatme, \& N. Ayanian, (2017, May). Crazyswarm: A large nano-quadcopter swarm. In Robotics and Automation (ICRA), 2017 IEEE International Conference on (pp. 3299-3304). IEEE.

6. M. W. Mueller, (2018, December). "A Dynamics-Agnostic State Estimator for Unmanned Aerial Vehicles Using Ultra-Wideband Radios," ASME 2018 Dynamic Systems and Control Conference, American Society of Mechanical Engineers Digital Collection.

7. J. L. Piquero, V. K. Delica, A. L. Orquia, E. M. Reynaldo, J. Ilao, M. A. Roque, ... \& H. Jayakody, (2019), "A NEW SLIDING MODE CONTROLLER IMPLEMENTATION ON AN AUTONOMOUS QUADCOPTER SYSTEM," International Journal of Automation and Smart Technology, 9(2), 53-63.

8. S. Fahandezh-Saad, \& M. W. Mueller, (2018, June). "An algorithm for real-time restructuring of a ranging-based localization network," International Conference on Unmanned Aircraft Systems (ICUAS) 2018 (pp. 236-242), IEEE.

9. "Loco Positioning System," bitcraze, n.d. [Online]. Available: https://www.bitcraze.io/loco-pos-system/. [Accessed: June 27, 2019].

10. "Loco Positioning Protocol," bitcraze, 2019. [Online]. Available: https://www.bitcraze.io/docs/lps-node-firmware/master/lpp/. [Accessed: June 28, 2019].

11. "Loco Positioning TDoA principles," bitcraze, 2019. [Online]. Available:https://www.bitcraze.io/docs/lps-node-firmware/master/tdoa _principles/. [Accessed September 17, 2019].

12. S. Dädeby and J. Hesselgren, "A system for indoor positioning using ultra-wideband technology," Masters dissertation, Dept. of Computer Science and Engineering, Chalmers University of Technology and University of Gothenburg, Gothenburg, Sweden, 2017.

13. "Crazyflie 2.1 | Bitcraze," bitcraze, 2019. [Online]. Available: https://www.bitcraze.io/crazyflie-2-1/. [Accessed: September 10, 2019].

14. "Loco Positioning Node | Bitcraze," bitcraze, 2019. [Online]. Available: https://www.bitcraze.io/loco-pos-node/. [Accessed: September 10, 2019].

15. "Loco Positioning Deck | Bitcraze," bitcraze, 2019. [Online]. Available: https://www.bitcraze.io/loco-pos-deck/. [Accessed: September 10, 2019].

\section{AUTHORS PROFILE}

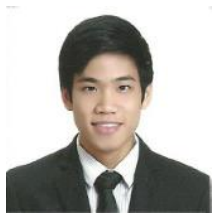

Timothy Chu obtained his BSME at De La Salle University (DLSU)-Manila, Philippines. He is currently taking up MSME at De La Salle University (DLSU)-Manila, Philippines and in Liverpool Hope University (LHU) in the United Kingdom.

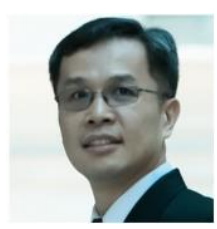

Alvin Chua is an Associate Professor of the Mechanical Engineering Department of De La Salle University, Philippines. He earned his BSME, MSME, and Ph.D. in ME at De La Salle University-Manila. As a scholar under the Department of Science and Technology-Engineering and Science Education Project (DOST-ESEP), he conducted his dissertation research at the University of New South Wales, Australia. He received a special citation for the 2003 NAST-DuPont Talent Search for Young Scientists (Mechanical Engineering). He has published several papers in international conferences like Conference on Decision and Control (CDC), and Advance Intelligent Mechatronics (AIM). His research interests are on mechatronics, robotics, optimal estimation, and controls.

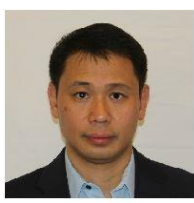

Edwin Sybingco is a faculty of the DLSU ECE Department. He completed his Doctoral of Philosophy in Electronics and Communications Engineering in De La Salle University area of Digital Signal Processing (DSP) focusing on Big Data and Intelligent Systems. He received his Master of Science (Electronics and Communications Engineering) from De La Salle University. He already published more than 30 scientific papers internationally in the field of DSP, Machine Vision, Computational Intelligence, and robotics.

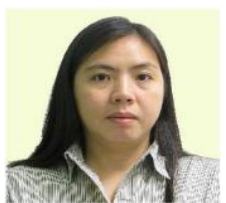

MA Antonette Roque is an Assistant Professor of the Electronics and Communications Engineering Department at De La Salle University, Manila, Philippines 1004. 